\title{
Light activation uncaged
}

Engineering cellular proteins with optical switches offers a powerful alternative to other methods of light-mediated cellular control.

The use of light to unlock 'caged' biologically active molecules is a powerful technique to specifically activate cellular pathways. Unfortunately, caged molecules show no receptor subtype selectivity, are depleted by uncaging and undesired photoproducts can be toxic. Such factors make them unsuitable for some in vivo studies.

But what if a photoswitchable agonist could be irreversibly tied to its target? This is a tactic that some researchers have been working on with increasing success. Now Ehud Isacoff and colleagues at the University of California in Berkeley and San Francisco show how such an optical switch can be used to precisely stimulate neurons (Szobota et al., 2007).

The technique relies on attaching agonists to extracellular receptors via an exogenously added photoisomerizable molecule that acts as a reversible optical switch. The agonist is covalently bound to a strategically placed cysteine on a mutated receptor using a reactive group on the optical switch. Last year the group engineered a light-gated ionotropic glutamate receptor channel (iGluR6) using a photoisomerizable cysteine-reactive glutamate called MAG (Volgraf et al., 2006).

iGluR6 is activated by glutamate binding resulting from photoisomerization of the glutamate-containing optical switch using 380-nm light. This depolarizes cells expressing the channel. Exposure to 500-nm light reverses the isomerization and closes the channel. In the present study, the authors show that iGluR6 allows temporally precise and rapid control of neuronal firing in transfected primary neurons; transgenic zebrafish expressing iGluR6 allowed reversible control of behavior.

MAG seems to have no adverse effect on the function of the target protein or other proteins in the cell. Isacoff says, "What's happening is this tiny photoswitch does calisthenics on the surface of the labeled protein as light goes on and off. But these molecular motions have no impact on protein function, unless the photoswitch is attached within about one nanometer of a ligand binding site. Then photoisomeriza- tion will permit the ligand to bind in one state and yank it out in the other state, producing reversible and selective activation."

Interest among neuroscientists in noninvasive methods to probe neural function has driven demand for light-based techniques, and this is not the first tool for activating neurons. "The natural thing to compare it to are the evolutionarily produced lightactivated proteins from bacteria such as channelrhodopsin-2 (ChR2) and halorhodopsin," comments Isacoff. "There is definitely an attraction to these opsins that use the natural chromophore retinal and may be able to pick it up endogenously in the host organism. MAG will always have to be added from outside," Isacoff acknowledges.

There are advantages to the photoswitch system though. Switching is faster, conductance levels are larger and more stable, and the on and off states of the switch are persistent, so you can flash a very brief light pulse $(\sim 1 \mathrm{~ms})$ and the resulting on or off state will be retained for many minutes. This would have advantages for behavioral analyses that could be confounded by continual light exposure like the touch-response experiments Isacoff performed in zebrafish. "This is an advantage you don't have with ChR2, which turns off rapidly when the light is extinguished," says Isacoff.

Another advantage of this method is generality. "If you have a ligand that can either disrupt or activate the function of a protein, in theory you should be able to generate a light-sensitive version that will work in vivo [using our system]," says Isacoff.

These are exciting times for neuroscientists. Proliferation of light-based methods for probing the brain provides opportunities and challenges. ChR2 is being rapidly embraced by the community, but it may not be the best choice for every application. As Isacoff says, "The exciting thing will be to try them side by side and see which works best. For studies of synaptic function there are advantages to gating a glutamate receptor derived from the organism under study."

\section{Daniel Evanko}

\section{RESEARCH PAPERS}

Szobota, S. et al. Remote control of neuronal activity with a light-gated glutamate receptor. Neuron 54, 535-545 (2007).

Volgraf, M. et al. Allosteric control of an ionotropic glutamate receptor with an optical switch. Nat. Chem. Biol. 2, 47-52 (2006). 